\title{
High Near-Field Enhancement in Plasmonic Coupled Nanostructure for Spaser Application
}

SAQIB JAMIL ( $\nabla$ engr.saqibjamil@gmail.com )

Sarhad University of Science and Information Technology https://orcid.org/0000-0003-3319-6065

Adnan Daud Khan

University of Engineering and Technology, Peshawar

Javed lqbal

Sarhad University of Science and Information Technology

Waqas Farooq

Sarhad University of Science and Information Technology

\section{Original Article}

Keywords: Nanostructure, Ellipse, Plasmon coupling, Symmetry breaking, Near-field enhancement, nano-elliptical dimer Posted Date: February 8th, 2021

DOI: https://doi.org/10.21203/rs.3.rs-162635/v1

License: (c) (i) This work is licensed under a Creative Commons Attribution 4.0 International License. Read Full License 


\section{Abstract}

We theoretically demonstrated a kind of plasmon coupled elliptical nanostructure to achieve a vast range of applications based on nanolaser or spaser with high intensity. To overcome the ohmic losses, the plasmon ellipse is composed of the gold film substrate with a gain media. A simple ellipse has been chosen from which variety of dimer configurations have been formed by symmetry alteration technique which are then tested for different light polarizations and gap variations. The proposed model supports localized surface plasmon resonance mode (LSPR). Moreover, the localized surface plasmon resonance (LSPR) property of the proposed nanostructure is numerically analysed by the finite-element method (FEM) and the results shows that the electric field intensity $(\mathrm{EFI})$ can be amplified to a large values by symmetry breaking in the elliptical nanostructure. Various plasmon modes can be excited by selecting the appropriate gain media. In addition to this, a compact tunable multi-wavelength nanolaser (spaser) can be developed by using this model. Giant near field enhancement (NFE) and high LSPR enable this structure to be promising for spaser applications such as surface enhanced Raman spectroscopy, sensing, lithography, imaging, dental applications and much more.

\section{Introduction}

Surface plasmon (SP) is actually the photon-excited collectively coherent oscillation of electrons at the surface of metallic nanostructures or films. Once the resonant interaction of surface charge oscillation and the electromagnetic field of the light has been realized, the restricted charges on the metal surface will dramatically enhance the electromagnetic field nearby and confine the electromagnetic energy into subwavelength regions, which exhibit strong resonant properties in optical spectra, namely surface plasmon resonance (SPR) [1]. The SPR reveals unique optical properties and spurs by new synthetic chemical methods of producing small particles in a variety of shapes quickly and inexpensively and advances micro/nanofabrication techniques [2-5], and has the prominent applications in surface-enhanced Raman scattering (SERS) [6, 7], optical sensing [8, 9], perfect light absorption [10-12], imaging and cloaking [13, 14], light speed manipulation [15-17], and alternative optical devices [18-24]. Nano-optics is now undergoing a period of explosive growth where new ideas, developments and impressive results appear literally on a daily basis. It is concerned with the science of concentrating optical energy into regions with subwavelength dimensions (typically tens of nanometres). Yet despite all this progress, there is still the need for a coherent, intense, ultrafast (with pulse durations down to a few femtoseconds), source of optical energy concentrated to nanoscale areas, similar to the laser but on a much smaller scale. Despite, all this heating problem that is associated with nanoparticles is also an issue of great concern. Since interaction of light with nanosized particles produces huge amount of heat due to metal presence in the structures which causes ohmic losses and suffers the performance of the systems so for this reason [25] proposed such a source that is based on surface plasmons so-called spaser (surface plasmon amplification by stimulated emission of radiation) and researchers are now working to develop and exploit this idea. Conventional semiconductor lasers are subject to the diffraction limit, and so their size cannot be less than half a wavelength, hindering the miniaturization of lasers [26]. Unlike conventional lasers, surface plasmon (SP)-based nanolasers can reduce the mode size and physical size of lasers to less than half a wavelength simultaneously, forming a nanoscale coherent light source far beyond the diffraction limit [27]. In order to achieve a lower threshold and lower loss transmission, a variety of nanolaser structures have been designed, which can be generally classified into metal-dielectric-metal structure nanolasers, metal nanoparticles plasmonic nanolasers [28-31], nanowire surface plasmonic nanolasers, array nanowires plasmonic nanolasers, W-G mode plasmonic nanolasers, etc $[32,33]$. Besides all this complex nanostructures have been deployed for investigating resonance phenomena, and those structures are much difficult to fabricate for practical realization of a model.

For observation of multiple resonances a dimer based on identical and mismatched gold ellipses, which are highly structure and polarization-dependent nanostructures. The nanostructures are wrapped by a gain media layer like silica and for simplicity we have set its value to 2.25 . The mode interactions with distinct angular momentums and line shapes are stronger in the asymmetric plasmonic nanodimers. Unlikely with homodimers, the symmetry reduced nanostructures sidesteps the crossings of the plasmonic modes resulting in a sharp resonance in the spectrum and possess better sensitivity than the symmetric

Page $2 / 21$ 
correspondents. The resonance observed in the mismatched nano-elliptical dimer (NED) can be enhanced and tuned by varying the distance between two nano-ellipses or modifying the size of one of the nano-ellipse. Here, we elucidate the results for the dimer separations only down to $3 \mathrm{~nm}, 5 \mathrm{~nm}, 7 \mathrm{~nm}, 9 \mathrm{~nm}$ and $11 \mathrm{~nm}$ because for such distances, the quantum mechanical effect, such as tunnelling of electrons through dimer junctions alters the classical response. Due to high polarization sensitivity of the proposed NE dimer, we have different response for $x$-y polarizations, therefore the dimer can be used as polarization sensitive beam filter [34]. Furthermore, we have spectral shape of lines with Fano resonance, which is thought to be a key element for high dispersive metamaterials and this is engineered through asymmetric spectral lines with steep variations.

\section{Model}

In this paper, we investigate various patterns of elliptical dimers formed from a single nano-ellip by scratching a portion and then splitting to form a nano-elliptical dimer (NED). This arrangement is done with special interest for the formation of spectral shapes of line. We found some arrangements that seem to be much beneficial for spectral line shapes and near-field enhancement (NFE). Polarization tuning and strong asymmetric spectral line shapes make the proposed NED an ideal test bed for spaser applications like switching, plasmon-induced transparency and SERS etc. The optical properties of the NEDs are carried out using COMSOL with RF module, while Johnson and Christy data model have been utilized for finding the dielectric constant value of gold [35] with air as an embedding medium. Figure 1 shows the schematic diagram of a heterodimer. The dimensions of the NED are $a_{1}, b_{1} / a_{2}, b_{2}$, where ' $a_{1}$ ' and ' $b_{1}$ ' are the semi-axis of one nanoellipse (NE), and ' $a_{2}$ ' and ' $b_{2}$ ' are the semi-axis of second nanoellipse (NE), with ellipse thickness' $t$ ' respectively. The gap between two nanoellipses is denoted by ' $S$ '. The illuminating electromagnetic wave is linearly polarized i.e. first we calculated the optical properties of NED for X-polarized case and then for y-polaroid one. Furthermore, we have broken the symmetry of one of the NE to transform the dimer for investigation of plasmonic responses and resonances.

\subsection{Optical Properties Of The Nano-elliptical Dimer (Ned)}

In this work, we examine different configurations of the nano-elliptical dimer (NED) structure, listed in Table 1.

Table 1 Various arrangements of a Nano-Elliptical Dimer (NED)

\begin{tabular}{|c|c|}
\hline Type & Geometry \\
\hline Type I & \\
\hline Type II & \\
\hline Type III & \\
\hline
\end{tabular}




\subsection{Type I Nano-Elliptical Dimer (NED)/Effect of polarization}

We analyze the optical properties of the "type I" dimer composed of two nanoellipses with dimensions; $\mathrm{a}_{1}=\mathrm{a} 2=100 \mathrm{~nm}, \mathrm{~b}_{1}=$ $b_{2}=60 \mathrm{~nm}, \mathrm{t}=35 \mathrm{~nm}$ with a separation $\mathrm{S}=3 \mathrm{~nm}$ respectively shown in Table 1 . Hot spots are formed in the regions where incident fields become concentrated and amplified. One more important parameter of a hot spot plane is a strong inhomogeneity of the incoming fields. We show in our case, the electrons are confined in two gap positions on the sharp edges of the nano-elliptical dimer and these charge electrons experience plasmonic field created by external illumination. In this dimer case the plasmon fields becomes non-uniform and greatly amplified at the edges due to the hot spots formation. Figure $2 \mathrm{a}$ depicts the extinction cross section obtained for $x$-y polarized cases, red line represents $x$-polarized case in which the light is efficiently coupled with the dimer. A small activation in the spectrum can be observed at $620 \mathrm{~nm}$ due to dark octupolar mode, along with a formation of Fano resonance, while a low energy peak at $688 \mathrm{~nm}$ appeared due to mixing of dipolar modes with octupolar modes. The peak at $859.9 \mathrm{~nm}$ shows the mixing of dipole and quadrupole modes and at the tail of this peak at 970.7 $\mathrm{nm}$ represents low energy bonding modes. A strong peak can be observed at $1381 \mathrm{~nm}$ due to hybridization of bright dipolar modes. The green line in the plot represents the y-polarized case in which a strong peak at $1155 \mathrm{~nm}$ can be seen and is of similar fashion as of x-polaroid version mainly due to combination bright dipolar modes that given birth to strong plasmonic effect and we attained two hot spots along with high energy spike. Another small rise can be seen at $739.9 \mathrm{~nm}$ mainly occurred due to dark modes. Figure 2(b-c) depicts the formation of hot spots and near field enhancement for $x$-y polarized cases respectively. It is visible that two hot spots were formed at the sharp edges of each NE, due to confinement of huge electric field between these two gap places. The near field enhancement for x-polarized NED was recorded to be 910 and for $y$-polaroid case this value reaches to almost 310 .

\subsubsection{Effect of the gap variation 'S' on the optical properties of Type I NED}

Here, we have examined the effect of gap variation 'S' on Type I NED while keeping all the other parameters fixed as described in Sect. 3.1. By giving variation in distance ' $S$ ' we have formed the same geometry as Type I and named the configurations as Type $I S_{3}$ for $S=3 \mathrm{~nm}$, Type $I S_{5}$ for $S=5 \mathrm{~nm}$, Type $I S_{7}$ for $S=7 \mathrm{~nm}$, Type $I S_{9}$ for $S=9 \mathrm{~nm}$ and Type $I S_{11}$ for $S=11 \mathrm{~nm}$ respectively. Figure $3 a$. depicts the extinction plot which shows the dependence of the hybridized plasmonic modes on different values of the gap region 'S'. For Type IS $S_{3}$ and Type $I S_{5}$ same type of mode hybridization occurred which can be clearly seen by red and bar type lines. A small rise appeared at about $620 \mathrm{~nm}$ due to dark octupolar modes followed by three consecutive heights at $659.5 \mathrm{~nm}$ (Type IS 7 ), $656.1 \mathrm{~nm}$ (Type IS 9 ) and $662.9 \mathrm{~nm}$ (Type IS ${ }_{11}$ ) represents mixing of dipolar and quadrupolar modes, similarly, simultaneous peaks at $688 \mathrm{~nm}$ gives the same nature. A narrow bonding dipole mode hybridization form peaks for all separation cases at about $850 \mathrm{~nm}$ to $860 \mathrm{~nm}$. At the vicinity of narrow and bright modes a height appeared due to mixing of quarupolar modes at $970.7 \mathrm{~nm}$ and the same point a Fano resonance can be noticed by purple curve.

A peak at $1234 \mathrm{~nm}$ for green and purple lines show strong bright dipolar modes, a slight red shifting is found at $1296 \mathrm{~nm}$ while the same peak is further red shifted and peak formed at $1381 \mathrm{~nm}$. Figure 3(b-f) depicts the formation of hot spots and near field enhancement (NFE) for $\mathrm{S}=3-11 \mathrm{~nm}$ respectively. The NFE values for each case recorded to be 910 (3nm and 5nm), 207, 191 and $188(7 \mathrm{~nm}, 9 \mathrm{~nm}$ and $11 \mathrm{~nm})$ respectively. A drastic drop in NFE value happened by increasing the gap between NEs which shows that NFE is highly sensitive to gap variation and is inversely proportional to the distance between the nanostructures. 


\subsubsection{Effect of symmetry breaking on the optical properties of Type I NED}

Metallic nanostructures support plasmon resonances whose energies depend sensitively on the geometrical shape of the structure. This tunability has stimulated considerable experimental and theoretical research [36]. An important topic in the field of plasmonics is the effect of symmetry breaking. For nanostructures that are small compared to the wavelength of incident light, only plasmons with finite dipole moments can be excited. For highly symmetric nanostructures such as a nanoshell, the symmetry breaking induced by a displacement of the dielectric core with respect to the metallic shell renders higher-order multipolar modes dipole active and therefore visible in the optical spectrum of the particle [37]. We have now engineered the Type I NED in such a way that size of one of the NE has been altered for various configurations. Here, we fixed the size of one $\mathrm{NE}$ as $\mathrm{a}_{1} / \mathrm{b}_{1} / \mathrm{t}=100 / 60 / 35 \mathrm{~nm}$ while the size of the other NE has been altered by different values to $\mathrm{a}_{2} / \mathrm{b}_{2} / \mathrm{t}=90 / 50 / 30$, $80 / 40 / 25,70 / 30 / 20,60 / 20 / 15$ and 50/10/10 nm to form Type IA, Type IB, Type IC, Type ID and Type IE respectively, while the separation between two NEs has been kept constant i.e. $\mathrm{S}=3 \mathrm{~nm}$ for each case (Fig. 4a). Figure $4 \mathrm{~b}$ depicts the extinction spectra for symmetry broken cases. The peaks at $1234 \mathrm{~nm}, 1188 \mathrm{~nm}, 1155 \mathrm{~nm}$ (Type IA and Type IB) and $1145 \mathrm{~nm}$ (Type IC, Type ID and Type IE) represents the hybridization of bright antibonding dipolar modes for each case respectively. The amplitude of the peaks drops sequentially according to the size decaying and the smallest height is shown by the green line. The peaks at $800.2 \mathrm{~nm}, 820.8 \mathrm{~nm}, 815.6 \mathrm{~nm}$ and $831.5 \mathrm{~nm}$ shows the strong hybridization of quadrupolar modes with same pattern of amplitude as their red shifted versions except for Type IE since it depicts broad antibonding dipolar spectra at 805.3 $\mathrm{nm}$ and $853.8 \mathrm{~nm}$. Mixing of octupolar modes occurs $652.8 \mathrm{~nm}$ except for blue line which occurred at $639.7 \mathrm{~nm}$. Fano resonances appeared at different wavelengths on the spectrum mainly due to dark octupolar modes at $853.8 \mathrm{~nm}, 831.5 \mathrm{~nm}$, $859.7 \mathrm{~nm}, 970.7 \mathrm{~nm}, 1009 \mathrm{~nm}$ and1025 nm. Figure 4(c-g) gives the charge distribution or near field enhancement (NFE) for Type (IA, IB, IC, ID and IE) respectively. The NFE value for each case is 171, 116, 163, 129 and 114. Once again close sized NED i.e. Type IA has the maximum value due to efficient coupling of light with the nano-structure which energized bright antibonding modes along with other higher order modes. Similarly, the value decreases with decreasing the size of one of the NE except for Type IB mainly due to activation of dark modes.

Table 2

Summary of various parameters for Type I NED

\begin{tabular}{|c|c|c|c|c|c|c|c|c|c|c|c|}
\hline $\begin{array}{l}\text { Structure } \\
\text { Type }\end{array}$ & Type I (x) & $\begin{array}{l}\text { Type } \\
\text { I (y) }\end{array}$ & $\begin{array}{l}\text { Type } \\
\text { IS }_{3} / I_{5}\end{array}$ & $\begin{array}{l}\text { Type } \\
I_{7}\end{array}$ & $\begin{array}{l}\text { Type } \\
I_{9}\end{array}$ & $\begin{array}{l}\text { Type } \\
I_{11}\end{array}$ & $\begin{array}{l}\text { Type } \\
\text { IA }\end{array}$ & $\begin{array}{l}\text { Type } \\
\text { IB }\end{array}$ & $\begin{array}{l}\text { Type } \\
\text { IC }\end{array}$ & $\begin{array}{l}\text { Type } \\
\text { ID }\end{array}$ & $\begin{array}{l}\text { Type } \\
\text { IE }\end{array}$ \\
\hline $\begin{array}{l}\text { No. of } \\
\text { Peaks }\end{array}$ & 4 & 2 & 4 & 3 & 4 & 3 & 5 & 6 & 6 & 7 & 7 \\
\hline Wavelength & 688 & 739.9 & 688 & 695.5 & 656.1 & 662.9 & 639.7 & 621 & 762 & 656.1 & 652.8 \\
\hline \multirow[t]{6}{*}{$(\mathrm{nm})$} & 859.9 & 115 & 859.5 & 853.8 & 831.5 & 842.5 & 800.2 & 649.4 & 815.6 & 719 & 748.5 \\
\hline & 970.7 & & 970.7 & 1296 & 956.2 & 1234 & 853.8 & 753 & 865.4 & 831.5 & 766.5 \\
\hline & 1381 & & 1381 & & 1234 & & 1025 & 820.8 & 970.7 & 895.7 & 805.3 \\
\hline & & & & & & & 1234 & 963.4 & 1009 & 1001 & 853.8 \\
\hline & & & & & & & & 1188 & 1155 & 1051 & 949 \\
\hline & & & & & & & & & & 1145 & 1155 \\
\hline NFE & 910 & 310 & 910 & 207 & 191 & 188 & 171 & 116 & 163 & 129 & 114 \\
\hline
\end{tabular}

\subsection{Type II Nano-Elliptical Dimer (NED)/Effect of polarization}


Next, we have engineered type II NED by keeping all the parametric values same as that of type I, instead we have rotated one ellipse by an angle $\theta=180^{\circ}$ to form a type II NED as shown in Table 1 for detailed examination of optical properties for $x-y$ polarization cases, effects of gap variation and symmetry breaking. The type II NED is an ideal test bed for studying because it supports multipolar progression, light polarization and dark plasmonic modes that can be spectrally tuned by simple incident polarization axis. Type II offers the possibility for dark plasmon to spectrally overlap a bright mode which strengthens the plasmonic effect. Furthermore, the designed model provides both even (dark) and odd (bright) parity plasmons to be transformed to interact with each other. In Fig. 5a we show the type II extinction plot along $x$-y position. The case contain contributions from all angular momentums resulted in the generation of five peaks for x-polarized luminance and three peaks for $y$-polarized luminance. The peak formed at $1494 \mathrm{~nm}$ shown by blue line is highly red shifted and is mainly due to the negative parity of dipoles as clear from its height. However, peaks at $1199 \mathrm{~nm}$ and $865.4 \mathrm{~nm}$ are formed due to strong hybridization of positive parities luminous modes that strongly interacted with even dark modes and given birth to these high energy peaks. The peak at $680.6 \mathrm{~nm}$ is blue shifted but still at good height level compared to the peak appeared at $1001 \mathrm{~nm}$. Similarly, y-polarized case show a red shifted peak at $1381 \mathrm{~nm}$ appeared due to mixing of dipolar modes of both nano-ellipses. The other low heighted peaks raised at $921.6 \mathrm{~nm}$ and $699.3 \mathrm{~nm}$ respectively due to poor light luminance resulting in the mixing of dark quadrupolar modes. Figure 5(b-c) shows the near field enhancements for $x-y$ polarized cases respectively and it is visible that charge distribution for $x$-polarized case is much better than y polarized case. The charges are distributed in the gap positions and along the surface of each nanoellipse (NE). Hot spots are formed at four different points, two at the tips while two in between gaps that produced huge plasmonic effect resulting in the near field enhancement (NFE) of about 238. Similarly, y-polarized case shows relatively less charge distribution due to weaker hybridization resulted in near field enhancement of about 110 .

\subsubsection{Effect of the gap variation 'S' on the optical properties of Type II NED}

Here, we have introduced separation ' $S$ ' in the Type II NED while keeping all the other parameters fixed as described in Sect. 3.1. By giving variation in distance ' $S$ ' we have formed the same geometry as Type II and named the configurations as $T_{y p e} I I S_{3}$ for $S=3 \mathrm{~nm}$, Type $\| S_{5}$ for $S=5 \mathrm{~nm}$, Type $\| S_{7}$ for $S=7 \mathrm{~nm}$, Type $\|_{9}$ for $S=9 \mathrm{~nm}$ and Type $\| S_{11}$ for $S=11$ nm respectively. Figure 6a depicts the extinction spectrum for the Type II gap variation case in which Type $\|_{3} S_{3}$ and Type IIS ${ }_{11}$ gives the identical spectral line shape, small peak at $615 \mathrm{~nm}$ shows the dark octupolar modes and at the tail a Fano resonance appears, quadrupolar modes of both ellipses interact with each other and a resonance originated at $680.6 \mathrm{~nm}$ followed by a dark octupolar height at $775.9 \mathrm{~nm}$. Two high energy spikes appeared at $865.4 \mathrm{~nm}$ and $1199 \mathrm{~nm}$ due to hybridization of dipolar bright modes. A quadrupolar interaction generates a resonance at $1001 \mathrm{~nm}$ while a same nature peak red shifted peak appears at $1494 \mathrm{~nm}$. Type IIS , Type IIS $_{7}$ and Type IIS 9 have the same nature and interaction of quadrupolar modes can be seen at 711 $\mathrm{nm}, 707 \mathrm{~nm}, 699.3 \mathrm{~nm}, 1155 \mathrm{~nm}, 1177 \mathrm{~nm}$ and $1188 \mathrm{~nm}$ respectively, while the dipolar antibonding modes appears at 902.1 nm, $889.5 \mathrm{~nm}, 883.3 \mathrm{~nm}, 1323 \mathrm{~nm}, 1283 \mathrm{~nm}$ and $1296 \mathrm{~nm}$ respectively. Fano resonances for Type IIS ${ }_{3}$ and Type IIS ${ }_{11}$ is visible at $615 \mathrm{~nm}$ while for rest of the cases it appeared at $1042 \mathrm{~nm}$. Figures 6(b-f) depicts the near field enhancement (NFE), Type IIS 3 shows 237, Type IIS $S_{5}$ produced a value of 302. The maximum value of NFE obtained for gap variation case is 362 in Type IIS while for Type IIS 9 is 306 and Type IIS ${ }_{11}$ gives lowest value of 216 . Type $\|_{3} S_{3}$ and Type $\|_{11}$ has a very close NFE value, furthermore same is the case with Type $\|_{5}$ and Type $\|_{9} S_{9}$.

\subsubsection{Effect of symmetry breaking on the optical properties of Type II NED}

We have now introduced symmetry breaking in the Type II NED by varying the parameters of one nano-ellipse to different values. The size of one NE is set as $a_{1} / b_{1} / t=100 / 60 / 35 \mathrm{~nm}$ while the size of the second NE has given different values of; $\mathrm{a}_{2} / \mathrm{b}_{2} / \mathrm{t}=90 / 50 / 30,80 / 40 / 25,70 / 30 / 20,60 / 20 / 15$ and 50/10/10 nm to form Type IIA, Type IIB, Type IIC, Type IID and Type IIE 
respectively, while the gap between the nano-structure is fixed at $S=3 \mathrm{~nm}$ for each case, Fig. 7a shows such scheme. Figure $7 \mathrm{~b}$ depicts the extinction spectra for symmetry broken cases. Type IIA shows same number of heights as of Type IIS 3 and Type IIS ${ }_{11}$ with bright antibonding modes at $1096 \mathrm{~nm}$ and $1168 \mathrm{~nm}$, broad quadrupolar hybridization formed peaks $810 \mathrm{~nm}$ and 790 $\mathrm{nm}$. The hybridization of octupolar modes is visible at $659.5 \mathrm{~nm}$ while a Fano resonance formed at $810.4 \mathrm{~nm}$. The bright dipolar and quadrupolar modes of Type IIB are stronger compared to Type IIC, Type IID and Type IIE. Another quadrupolar peak for Type IIE appeared at $753 \mathrm{~nm}$. Dark octupolar modes spectra for all cases can be seen in the range of 603-650 nm with Fano resonances at the tails of Type IIB and Type IIC.

Figure 7 (a) Schematic of symmetry broken Type II NED (b) Extinction Cross-section of simple symmetry broken Nano-elliptical dimer (NED) type II for x-polarization, (c-g) Near Field Enhancement (NFE) of symmetry broken type II NED for x-polarization luminance.

Table 3

Summary of various parameters for Type II NED

\begin{tabular}{|c|c|c|c|c|c|c|c|c|c|c|c|}
\hline $\begin{array}{l}\text { Structure } \\
\text { Type }\end{array}$ & Type II (x) & $\begin{array}{l}\text { Type } \\
\text { II (y) }\end{array}$ & $\begin{array}{l}\text { Type } \\
\text { IIS }_{3} / \|_{11}\end{array}$ & $\begin{array}{l}\text { Type } \\
\text { IIS }_{5}\end{array}$ & $\begin{array}{l}\text { Type } \\
\text { IIS }_{7}\end{array}$ & $\begin{array}{l}\text { Type } \\
\text { IIS }_{9}\end{array}$ & $\begin{array}{l}\text { Type } \\
\text { IIA }\end{array}$ & $\begin{array}{l}\text { Type } \\
\text { IIB }\end{array}$ & $\begin{array}{l}\text { Type } \\
\text { IIC }\end{array}$ & $\begin{array}{l}\text { Type } \\
\text { IID }\end{array}$ & $\begin{array}{l}\text { Type } \\
\text { IIE }\end{array}$ \\
\hline $\begin{array}{l}\text { No. of } \\
\text { Peaks }\end{array}$ & 5 & 3 & 7 & 8 & 7 & 5 & 5 & 5 & 6 & 6 & 4 \\
\hline Wavelength & 680.6 & 699.3 & 615 & 618 & 621 & 609.1 & 659.5 & 652.8 & 612 & 603 & 649.4 \\
\hline \multirow[t]{7}{*}{$(\mathrm{nm})$} & 865.4 & 921.6 & 680.6 & 656.1 & 659.5 & 699.3 & 800.2 & 780.6 & 649.4 & 649.4 & 753 \\
\hline & 1001 & 1381 & 775.9 & 711 & 707 & 883.3 & 837 & 820.8 & 753 & 707 & 826.1 \\
\hline & 1199 & & 865.4 & 842.5 & 889.5 & 1155 & 1096 & 963.4 & 820.8 & 826.1 & 1135 \\
\hline & 1494 & & 1001 & 902.1 & 1042 & 1296 & 1168 & 1146 & 935.1 & 1034 & \\
\hline & & & 1199 & 993.5 & 1188 & & & & 1155 & 1155 & \\
\hline & & & 1494 & 1177 & 1283 & & & & & & \\
\hline & & & & 1323 & & & & & & & \\
\hline \multirow[t]{2}{*}{ NFE } & 238 & 110 & $230 /$ & 302 & 362 & 302 & 196 & 113 & 96 & 110 & 140 \\
\hline & & & 216 & & & & & & & & \\
\hline
\end{tabular}

\subsection{Type III Nano Elliptical Dimer (NED)/Effect of polarization}

In this section, we investigate the optical properties of a type III NED. The building parameters are kept constant as that of type I and II. Only an axial rotation of $\theta=180^{\circ}$ is given to one nano-ellipse to form a Type III NED as shown in Table 1 . This configuration is the one in which the single tip side of each NE face each other with identical dimensions forming a nanoantenna known as "bowtie antenna". The extinction spectra of such configuration for x-y polarization is shown in Fig. 8 a. Blue line in the spectrum represents $x$-polaroid case for which the strong peak appears at $1059 \mathrm{~nm}$ due to efficient coupling of light with the bowtie antenna resulting in the activation of even parities which causes the hybridization of dipolar-dipolar bonding modes. After this the lines goes down and a small height appeared at $805.3 \mathrm{~nm}$ due to antisymmetric alignment of the weak quadrupolar mode. Similarly, for y-polarization the dark modes were not actively reacted with bright modes resulted in the low height red shifted peak occurred at the same position as that of $\mathrm{x}$-axis at the wavelength of $1059 \mathrm{~nm}$ but shows less energy. Another peak appears which basically represents dark modes at $739.9 \mathrm{~nm}$. Furthermore, a little spike can be observed 
at $597.7 \mathrm{~nm}$ which also shows the poor interaction of light with the bowtie antenna compared to x-polaroid case. These peaks can be tuned by slight geometrical alteration or varying the gap and this is highly suitable for high sensitive biosensors [36]. Figure $8(\mathrm{~b}-\mathrm{c})$ corresponds to surface charge distributions, the surface charge distributions are strongly bonded with incident light polarization. Strong correlation between geometry and local field enhancement can be observed. The mapping clearly shows the fundamental dipole mode for each NE, and hybridized dipolar mixing of charges. A strong coupling occurred when the light was incident from $x$-axis due to which hot spot can be clearly seen between the gaps of the two nano-structures. This point reveals the strong antibonding modes that led to grow the near field enhancement value up to 492 . Furthermore, $y$ polarized map shows poor coupling due to which bonding modes were formed and unable to activate dark modes. Also, the charge distributions were mainly formed on the sharp edges of the nano-structures and clear hot spot was not formed between the gaps of the dimer. NFE for this case was found to be about 70 which is almost 7 times less than the $x$-polarized case.

\subsubsection{Effect of the gap variation ' $S$ ' on the optical properties of Type III NED}

In this section we have brought gap variation ' $S$ ' in the Type III NED while fixing all the parameters same as of Sect. 3.1. By varying the separation ' $S$ ' a same Type III geometry has been formed and given names for simplicity such as Type IIIS ${ }_{3}$ for $S=$

$3 \mathrm{~nm}$, Type IIIS $S_{5}$ for $S=5 \mathrm{~nm}$, Type IIIS $S_{7}$ for $S=7 \mathrm{~nm}$, Type IIIS 9 for $S=9 \mathrm{~nm}$ and Type $I I \mathrm{~S}_{11}$ for $\mathrm{S}=11 \mathrm{~nm}$ respectively. Figure $9 \mathrm{a}$ depicts the extinction spectrum for the Type III gap variation case in which all the cases shows almost identical line shape. Since, Type $\mathrm{III}_{3}$ has minimum separation so strong dipole-dipole hybridization forms a broad peak at $1059 \mathrm{~nm}$ and is more red shifted compared to other peaks. Another peak appeared due to the mixture of broad dipolar and quadrupolar modes at $805.3 \mathrm{~nm}$ and is again a slight red shifted compared to others and a Fano resonance can be noticed at $646.2 \mathrm{~nm}$ mainly due to dark octupolar modes. Same kind of hybridization occurred for other separation cases and peaks formed and almost similar fashion peaks appeared on the spectrum details given in Table 3. Figure 9(b-f) provides the formation of spots and near field enhancement for gap variations. Once again NFE value shows a close relationship with the separation and the highest NFE value is obtained from Type IIIS 3 which goes up to 492 followed by a drastic drop in NFE value to 107 for Type IIIS 5 . Remaining NFE values are listed in Table 3.

\subsubsection{Effect of symmetry breaking on the optical properties of Type III NED}

The parameters are set same as Sect. 3.2.2 while the geometry followed is Type III, such configuration is shown in Fig. 10a. Figure 10b shows the extinction spectrum in which Type IIIA shows bright dipolar-dipolar mode mixing at $1145 \mathrm{~nm}$ and 956,2 $\mathrm{nm}$ while octupolar hybridization for same case produced low energy spikes at $748.5 \mathrm{~nm}$ and $815.6 \mathrm{~nm}$. Type IIIB and Type IIIC shows same nature of peaks while Type IIID shows octupolar mixing at $703.1 \mathrm{~nm}$, quadrupolar hybridization at $790.3 \mathrm{~nm}$ and $1059 \mathrm{~nm}$ with dipole-dipole mode formation at $949.1 \mathrm{~nm}$. Type IIIE shows octupolar hybridization at $612 \mathrm{~nm}, 723.1 \mathrm{~nm}, 805.1$ $\mathrm{nm}$ and $1125 \mathrm{~nm}$, while dipolar-dipolar mixing formed a peak at $935.1 \mathrm{~nm}$. Similarly, Fig. 10(c-g) gives the formation of hot spots and NFE. The highest value of NFE is shown by Type IIIA since it has the minimum separation due to which effective plasmonic mixing occurred which resulted a high value. Further achieved NFE values are listed in Table 4. 
Table 4

Summary of various parameters for Type III NED

\begin{tabular}{|c|c|c|c|c|c|c|c|c|c|c|c|c|}
\hline $\begin{array}{l}\text { Structure } \\
\text { Type }\end{array}$ & $\begin{array}{l}\text { Type } \\
\text { III (x) }\end{array}$ & $\begin{array}{l}\text { Type } \\
\text { III (y) }\end{array}$ & $\begin{array}{l}\text { Type } \\
\text { IIIS }_{3}\end{array}$ & $\begin{array}{l}\text { Type } \\
\text { IIIS }_{5}\end{array}$ & $\begin{array}{l}\text { Type } \\
\mathrm{IIS}_{7}\end{array}$ & $\begin{array}{l}\text { Type } \\
\text { IIIS }_{9}\end{array}$ & $\begin{array}{l}\text { Type } \\
\text { IIIS }_{11}\end{array}$ & $\begin{array}{l}\text { Type } \\
\text { IIIA }\end{array}$ & $\begin{array}{l}\text { Type } \\
\text { IIIB }\end{array}$ & $\begin{array}{l}\text { Type } \\
\text { IIIC }\end{array}$ & $\begin{array}{l}\text { Type } \\
\text { IIID }\end{array}$ & TypellIE \\
\hline $\begin{array}{l}\text { No. of } \\
\text { Peaks }\end{array}$ & 2 & 3 & 2 & 2 & 2 & 2 & 2 & 3 & 3 & 3 & 3 & 4 \\
\hline Wavelength & 805.3 & 597.7 & 805.3 & 775.9 & 757.4 & 753 & 753 & 815.6 & 800.2 & 810.4 & 790.3 & 723.1 \\
\hline \multirow[t]{3}{*}{$(\mathrm{nm})$} & 1059 & 739.9 & 1059 & 1001 & 970.7 & 970.7 & 978.2 & 956.2 & 963.4 & 956.2 & 949.1 & 805.3 \\
\hline & & 1059 & & & & & & 1145 & 1096 & 1077 & 1059 & 935.1 \\
\hline & & & & & & & & & & & & 1125 \\
\hline NFE & 492 & 70 & 490 & 107 & 121 & 138 & 112 & 492 & 228 & 313 & 136 & 223 \\
\hline
\end{tabular}

Figure 11 gives a comparison for the best case amongst all the discussed cases above in terms of NFE and multiple wavelength spectrum range. Type I $(x)$ i.e. when light was incident on the Type I NED from $x$-direction so it produced a huge NFE value of about 910 which is highest value recorded for this study, along with a vast range of operational wavelength with 4 peaks. Type II (x) produced a NFE value of about 238 but with a highest wavelength range with 5 peaks. Type III (x) generated a NFE value of about 492 but with only 2 peaks along the wavelength spectrum.

\section{Conclusion}

We have investigated the generation of higher order plasmonic modes in a simple elliptical structure, its possible symmetry broken versions and gap variations illuminated by a linear x-y polarized light. For a simple Type I NED uniform charge distribution along with the formation of hot spot was found with a good line shape of extinction spectra. Same parameters were applied to a Type II and III NED structure which caused an efficient coupling of dark quadrupolar modes with bright dipolar modes creating multiple peaks in the spectrum. Then we performed symmetry breaking on Type I,II and III respectively, since symmetry breaking technique is an ideal methodology for performance enhancement layout. This introduced numerous heights at different wavelengths due to strong mixing of even and odd modes. We extended our findings to form symmetry breaking and gap variations with an axial rotations from $\theta=0^{\circ}-180^{\circ}$ that produced variants of NED configuration. This introduced multi-wavelengths starting from two peaks to seven peaks along with giant NFE for x-polaroid along with its variant cases. Moreover, Type I and its variants produced highest NFE followed by Type III. While Type II and its sub-classes produced relatively small NFE values. To, conclude Type I, II, III along with their variants are ideal candidates for producing pronounced tunable resonances and higher order dark modes in the visible and near infrared regions, which may be useful for number of spaser applications like electromagnetic-induced transparency, slow light, switching, sensing, bio-medical and SERS applications.

\section{Declarations}

\section{Ethical Approval:}

All authors of this research paper have directly participated in the planning, execution, or analysis of this study.

1. All authors of this paper have read and approved the final version submitted.

2. The contents of this manuscript have not been copyrighted or published previously.

3. The contents of this manuscript are not now under consideration for publication elsewhere. 
4. The contents of this manuscript will not be copyrighted, submitted, or published elsewhere, while acceptance by the Journal is under consideration.

5. All sources used are properly disclosed (Correct citation). Literally copying of text must be indicated as such by giving proper references.

6. There are no directly related manuscripts or abstracts, published or unpublished, by any authors of this paper.

7. All authors have been personally and actively involved in substantial work leading to the paper, and will take public responsibility for its content.

Consent to Participate: Not applicable

Consent to Publish: Not applicable

Authors Contribution: S. Jamil, A. D. Khan, and J. Iqbal proposed the concept. S. Jamil performed the simulations. All authors analysed the data and discussed the results. S. Jamil and W. Farooq wrote the draft. W. Farooq edited the manuscript. J. Iqbal and A. D. Khan supervised the project.

Declarations: None

Funding completing interest: None

Conflicts of interest: The authors declare that they have no conflict of interest.

Availability of data and material: Already available in manuscript

Code: Will be available upon request

Acknowledgment: The authors would like to thank Sarhad University of Science and Information Technology (SUIT) and US Pakistan Center for Advanced Studies in Energy, University of Engineering \& Technology, Peshawar, 25000, Pakistan

\section{References}

1. E. L. Gool, I. Stojanovic, R. B. Schasfoort, A. Sturk, T. G. Van Leeuwen, R. Nieuwland, et al., "Surface plasmon resonance is an analytically sensitive method for antigen profiling of extracellular vesicles," Clinical chemistry, vol. 63, pp. 1633-1641, 2017.

2. X. Lu, M. Rycenga, S. E. Skrabalak, B. Wiley, and Y. Xia, "Chemical synthesis of novel plasmonic nanoparticles," Annual review of physical chemistry, vol. 60, pp. 167-192, 2009.

3. H. Xin, C. Fan, J. Wang, E. Liang, and S. Zhu, "Synthesis of indium-silver bimetallic nanocomposites for surface-enhanced Raman scattering," Optical Review, vol. 25, pp. 678-683, 2018.

4. D. Yuan, S. Chen, Y. Wu, and J. Wang, "A facile surface enhanced Raman scattering substrate based on silver deposited sandpaper," Modern Physics Letters B, vol. 33, p. 1950239, 2019.

5. N. C. Lindquist, P. Nagpal, K. M. McPeak, D. J. Norris, and S.-H. Oh, "Engineering metallic nanostructures for plasmonics and nanophotonics," Reports on Progress in Physics, vol. 75, p. 036501, 2012.

6. J. Z. Zhang and C. Noguez, "Plasmonic optical properties and applications of metal nanostructures," Plasmonics, vol. 3, pp. 127-150, 2008.

7. B. Yuan, W. Zhou, and J. Wang, "Novel H-shaped plasmon nanoresonators for efficient dual-band SERS and optical sensing applications," Journal of Optics, vol. 16, p. 105013, 2014. 
8. Z. Liu, G. Liu, G. Fu, X. Liu, Z. Huang, and G. Gu, "All-metal meta-surfaces for narrowband light absorption and high performance sensing," Journal of Physics D: Applied Physics, vol. 49, p. 445104, 2016.

9. J. Wang, C. Fan, J. He, P. Ding, E. Liang, and Q. Xue, "Double Fano resonances due to interplay of electric and magnetic plasmon modes in planar plasmonic structure with high sensing sensitivity," Optics express, vol. 21, pp. 2236-2244, 2013.

10. N. Liu, M. Mesch, T. Weiss, M. Hentschel, and H. Giessen, "Infrared perfect absorber and its application as plasmonic sensor," Nano letters, vol. 10, pp. 2342-2348, 2010.

11. J. Wang, C. Fan, P. Ding, J. He, Y. Cheng, W. Hu, et al., "Tunable broad-band perfect absorber by exciting of multiple plasmon resonances at optical frequency," Optics express, vol. 20, pp. 14871-14878, 2012.

12. C. Cen, Z. Yi, G. Zhang, Y. Zhang, C. Liang, X. Chen, et al., "Theoretical design of a triple-band perfect metamaterial absorber in the THz frequency range," Results in Physics, vol. 14, p. 102463, 2019.

13. Q. Ma, H. Hu, E. Huang, and Z. Liu, "Metamaterial based compressive spatial-spectral transformation microscope," in 2017 Conference on Lasers and Electro-Optics (CLEO), 2017, pp. 1-1.

14. X. Ni, Z. J. Wong, M. Mrejen, Y. Wang, and X. Zhang, "An ultrathin invisibility skin cloak for visible light," Science, vol. 349, pp. 1310-1314, 2015.

15. J. Wang, B. Yuan, C. Fan, J. He, P. Ding, Q. Xue, et al., "A novel planar metamaterial design for electromagnetically induced transparency and slow light," Optics Express, vol. 21, pp. 25159-25166, 2013.

16. P. Ding, J. He, J. Wang, C. Fan, and E. Liang, "Electromagnetically induced transparency in all-dielectric metamaterialwaveguide system," Applied Optics, vol. 54, pp. 3708-3714, 2015.

17. J. Wang, J. Zhang, C. Fan, K. Mu, E. Liang, and P. Ding, "Electromagnetic field manipulation in planar nanorod antennas metamaterial for slow light application," Optics Communications, vol. 383, pp. 36-41, 2017.

18. M.-X. Ren, W. Wu, W. Cai, B. Pi, X.-Z. Zhang, and J.-J. Xu, "Reconfigurable metasurfaces that enable light polarization control by light," Light: Science \& Applications, vol. 6, pp. e16254-e16254, 2017.

19. C. Cen, Y. Zhang, X. Chen, H. Yang, Z. Yi, W. Yao, et al., "A dual-band metamaterial absorber for graphene surface plasmon resonance at terahertz frequency," Physica E: Low-dimensional Systems and Nanostructures, vol. 117, p. 113840, 2020.

20. P. Ding, M. Li, J. He, J. Wang, C. Fan, and F. Zeng, "Guided mode caused by silicon nanopillar array for light emission enhancement in color-converting LED," Optics express, vol. 23, pp. 21477-21489, 2015.

21. H. Cheng, X. Wei, P. Yu, Z. Li, Z. Liu, J. Li, et al., "Integrating polarization conversion and nearly perfect absorption with multifunctional metasurfaces," Applied Physics Letters, vol. 110, p. 171903, 2017.

22. F. Lu, W. Zhang, L. Huang, S. Liang, D. Mao, F. Gao, et al., "Mode evolution and nanofocusing of grating-coupled surface plasmon polaritons on metallic tip," Opto-Electronic Advances, vol. 1, p. 180010, 2018.

23. P. Ding, Y. Li, L. Shao, X. Tian, J. Wang, and C. Fan, "Graphene aperture-based metalens for dynamic focusing of terahertz waves," Optics express, vol. 26, pp. 28038-28050, 2018.

24. M. Liu, F. Lu, W. Zhang, L. Huang, S. Liang, D. Mao, et al., "Highly efficient plasmonic nanofocusing on a metallized fiber tip with internal illumination of the radial vector mode using an acousto-optic coupling approach," Nanophotonics, vol. 8, pp. 921929, 2019.

25. D. J. Bergman and M. I. Stockman, "Surface plasmon amplification by stimulated emission of radiation: quantum generation of coherent surface plasmons in nanosystems," Physical review letters, vol. 90, p. 027402, 2003.

Page $11 / 21$ 
26. H.-P. Solowan and C. Kryschi, "Facile design of a plasmonic nanolaser," Condensed Matter, vol. 2, p. 8, 2017.

27. F. Wang, S. Ke, C. Qin, B. Wang, H. Long, K. Wang, et al., "Topological interface modes in graphene multilayer arrays," Optics \& Laser Technology, vol. 103, pp. 272-278, 2018.

28. Z. Torbatian and R. Asgari, "Plasmonic physics of 2D crystalline materials," Applied Sciences, vol. 8, p. $238,2018$.

29. S. Liu, F. Li, X. Han, L. Xu, F. Yao, and Y. Liu, "Preparation and Two-Photon Photoluminescence Properties of Organic Inorganic Hybrid Perovskites (C6H5CH2NH3) 2PbBr4 and (C6H5CH2NH3) 2Pbl4," Applied Sciences, vol. 8, p. $2286,2018$.

30. C. Huo, Z. Wang, X. Li, and H. Zeng, "Low-Dimensional Metal Halide Perovskites: a Kind of Microcavity Laser Materials," Chin. J. Lasers, vol. 44, pp. 120-131, 2017.

31. D. Zhang, Y. Xiang, J. Chen, J. Cheng, L. Zhu, R. Wang, et al., "Extending the propagation distance of a silver nanowire plasmonic waveguide with a dielectric multilayer substrate," Nano letters, vol. 18, pp. 1152-1158, 2018.

32. S. Wu, S. Buckley, J. R. Schaibley, L. Feng, J. Yan, D. G. Mandrus, et al., "Monolayer semiconductor nanocavity lasers with ultralow thresholds," Nature, vol. 520, pp. 69-72, 2015.

33. L. Zhou, "Study on Photoelectric Characteristics of Typical Graphene Structure Based on FDFD Method," ed: Anhui University Press: Anhui, China, 2015.

34. S. Mukherjee, H. Sobhani, J. B. Lassiter, R. Bardhan, P. Nordlander, and N. J. Halas, "Fanoshells: nanoparticles with built-in Fano resonances," Nano letters, vol. 10, pp. 2694-2701, 2010.

35. P. B. Johnson and R.-W. Christy, "Optical constants of the noble metals," Physical review B, vol. 6, p. 4370, 1972.

36. C. L. Nehl and J. H. Hafner, "Shape-dependent plasmon resonances of gold nanoparticles," Journal of Materials Chemistry, vol. 18, pp. 2415-2419, 2008.

37. Y. Wu and P. Nordlander, "Plasmon hybridization in nanoshells with a nonconcentric core," The Journal of chemical physics, vol. 125, p. 124708, 2006.

\section{Figures}




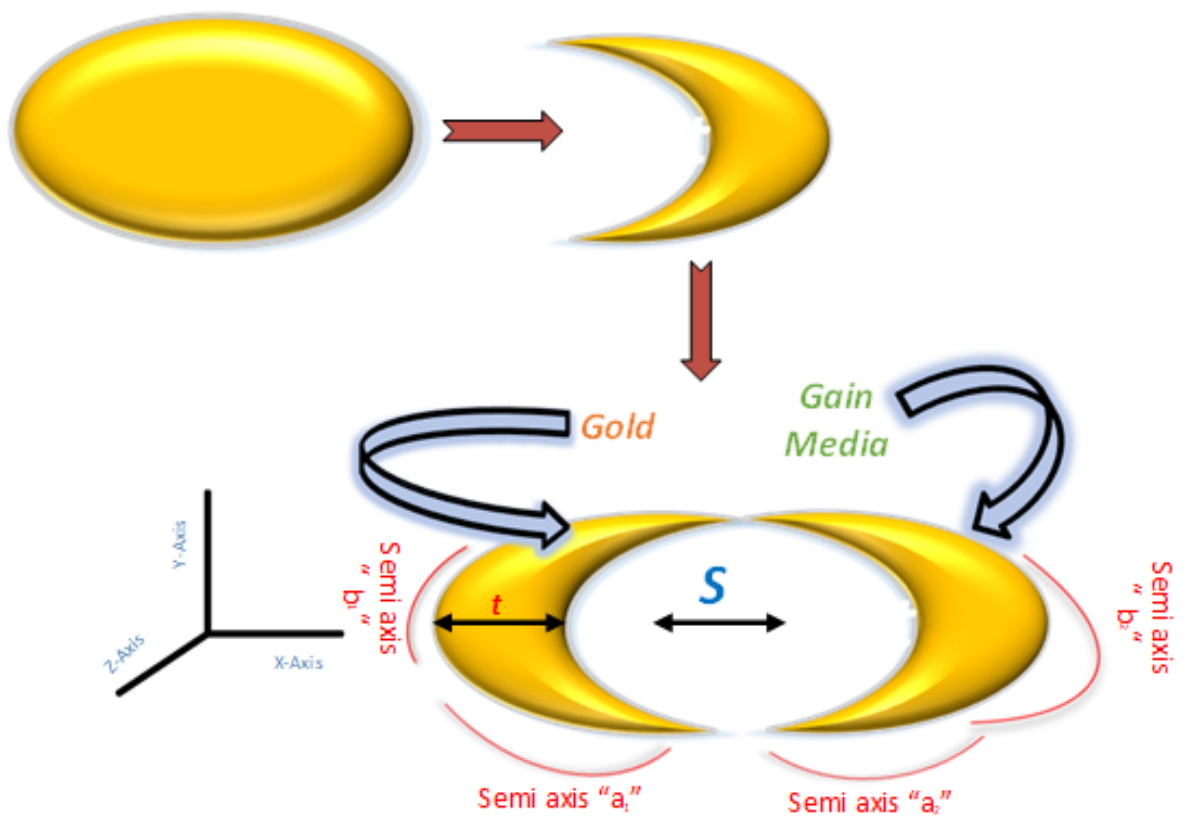

Figure 1

Transformation of single Nano-Ellipse (NE) to Nano-Elliptical Dimer (NED) with gain media layer

a.

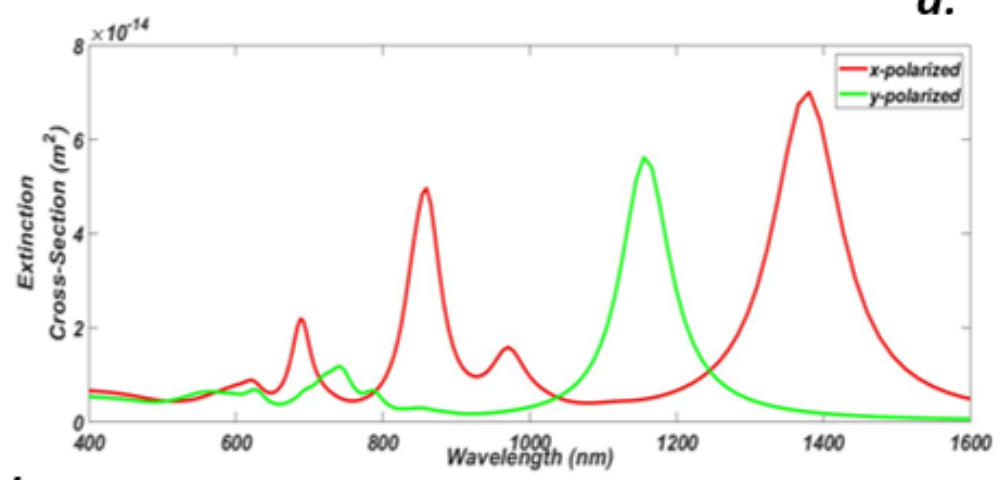

b.
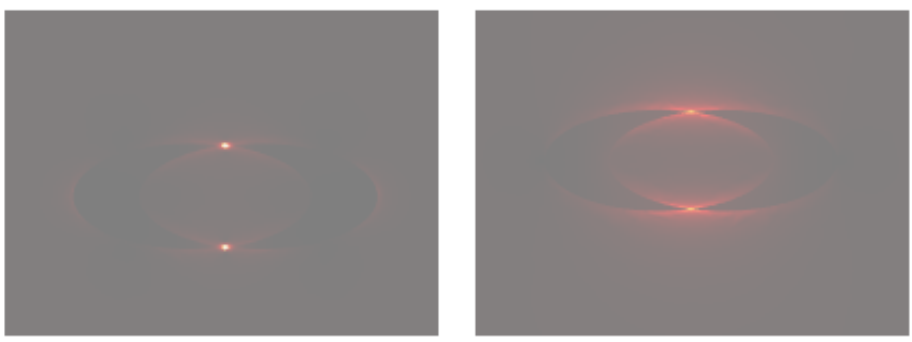

C.

$\mathbf{\nabla} 100$

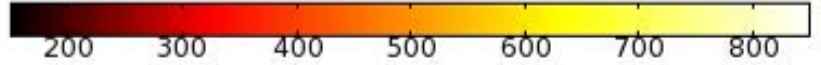

A 850

\section{Figure 2}

(a) Extinction Cross-section of simple Nano-elliptical dimer (NED) type I for $x$-y polarization, (b) Near Field Enhancement (NFE) of type I NED for x-polarization luminance, (c) Near Field Enhancement (NFE) of type I NED for y-polarization luminance. 


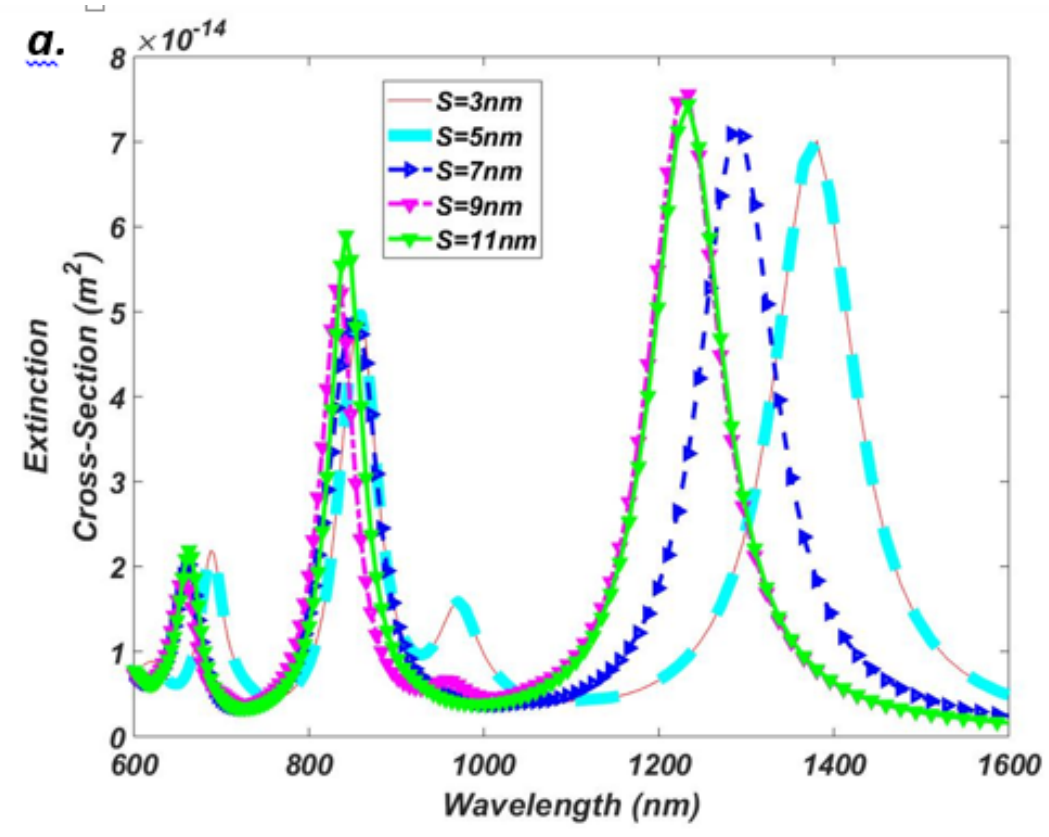

b.

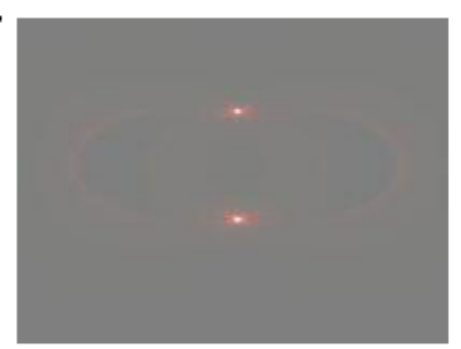

c.

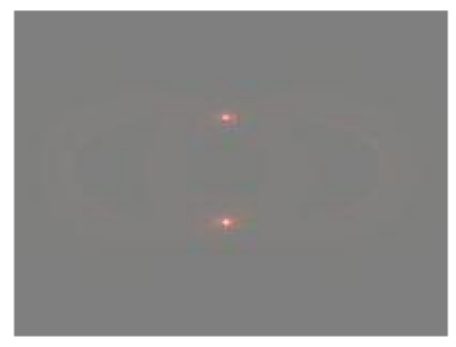

d.

e.

$f$.
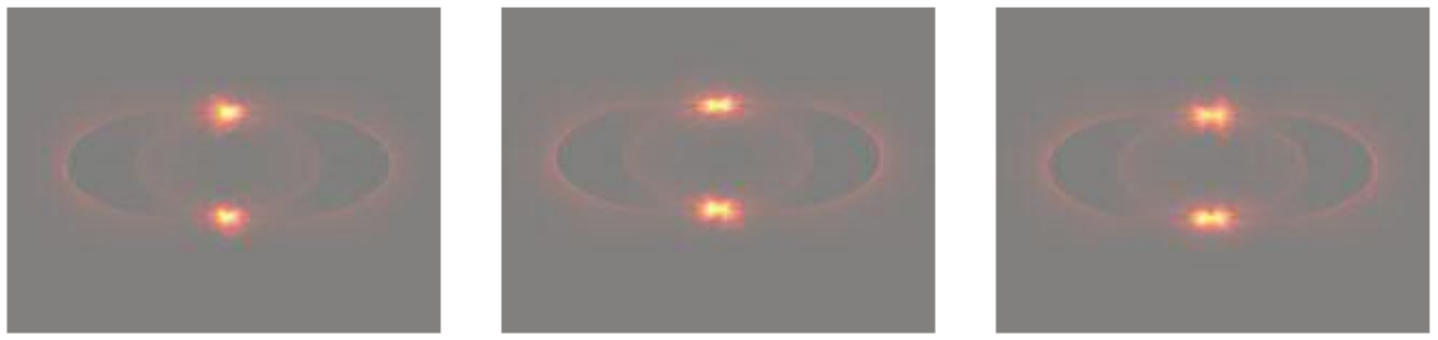

$\nabla 100$

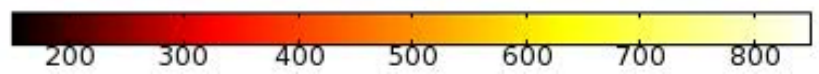

$\mathbf{\Delta} 850$

Figure 3

(a) Extinction Cross-section of simple Nano-elliptical dimer (NED) type I for gap variation along x-polarization, (b-f) Near Field Enhancement (NFE) of type I NED for x-polarization luminance with separation between nano-ellipses (NE) S=3nm, 5nm, 7nm, $9 \mathrm{~nm}$ and $11 \mathrm{~nm}$ respectively 

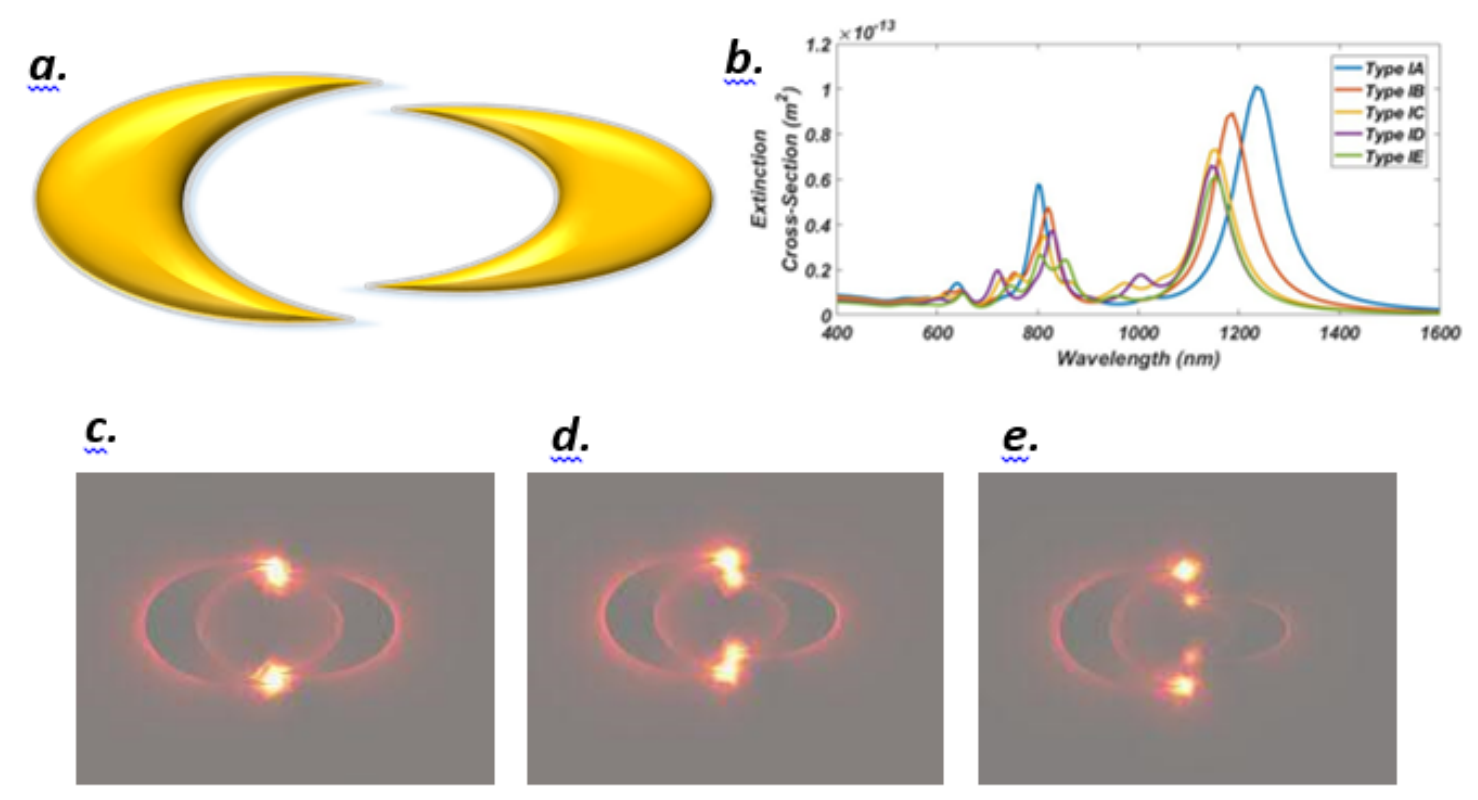

d.

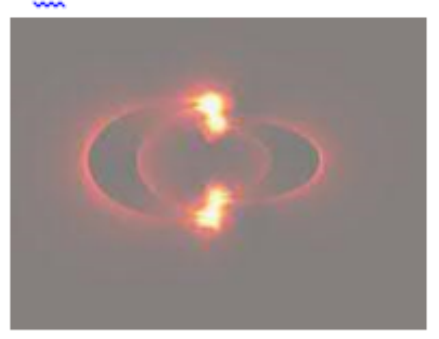

f.
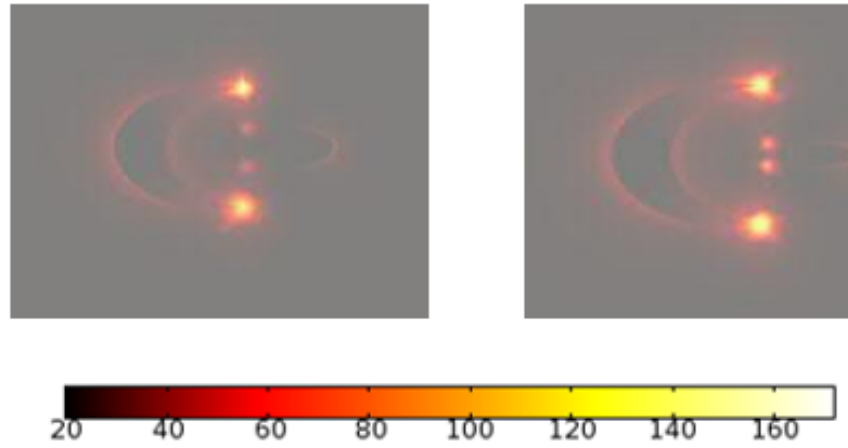

e.

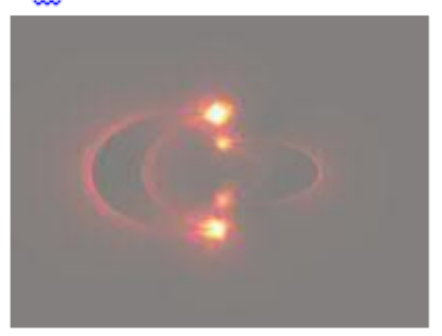

g.

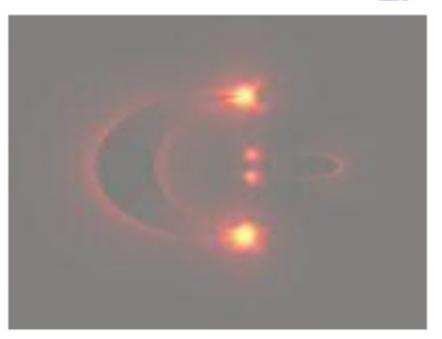

$\boldsymbol{\nabla} 20$

А 160

\section{Figure 4}

(a) Schematic of symmetry broken Type I NED (b) Extinction Cross-section of simple symmetry broken Nano-elliptical dimer (NED) type I for x-polarization, (c-g) Near Field Enhancement (NFE) of symmetry broken type I NED for x-polarization luminance. 


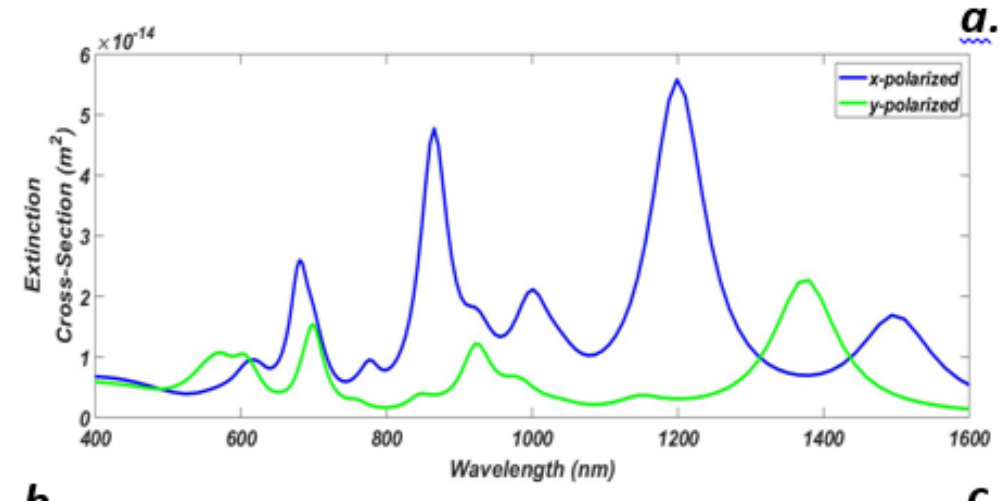

b.
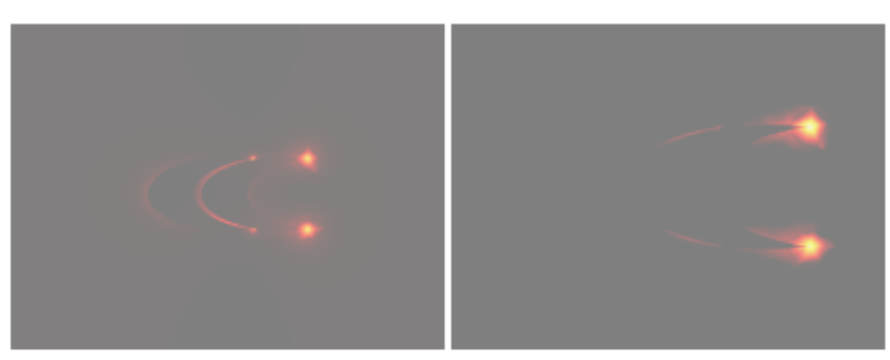

c.

$\boldsymbol{\nabla} 20$

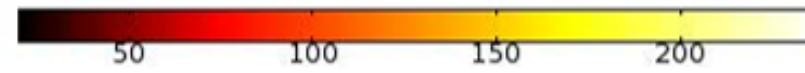

$\mathbf{\Delta} 220$

\section{Figure 5}

(a) Extinction Cross-section of simple Nano-elliptical dimer (NED) type II for $x$-y polarization, (b) Near Field Enhancement (NFE) of NED type II for x-polarization luminance, (c) Near Field Enhancement (NFE) of NED type II for y-polarization luminance. 

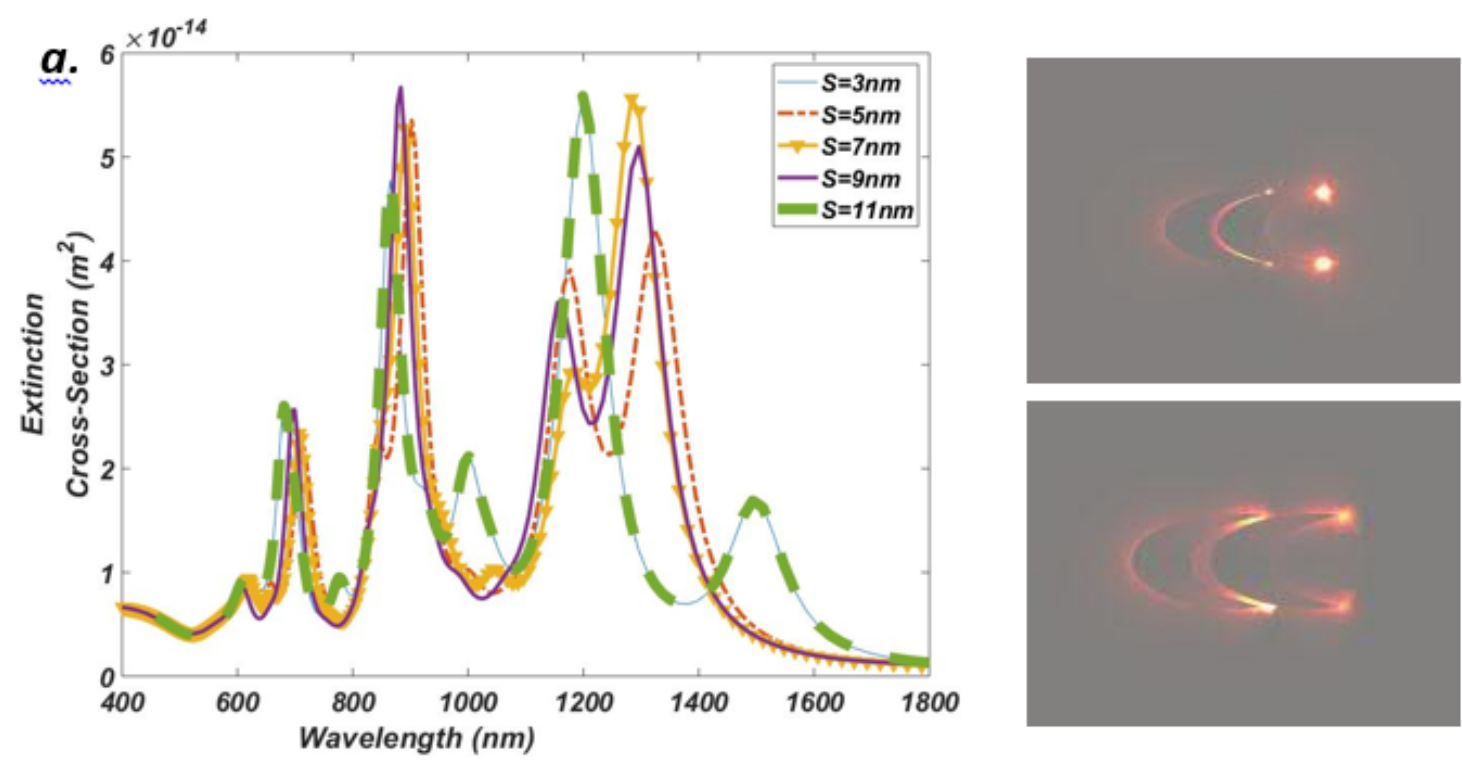

$\underline{b}$.
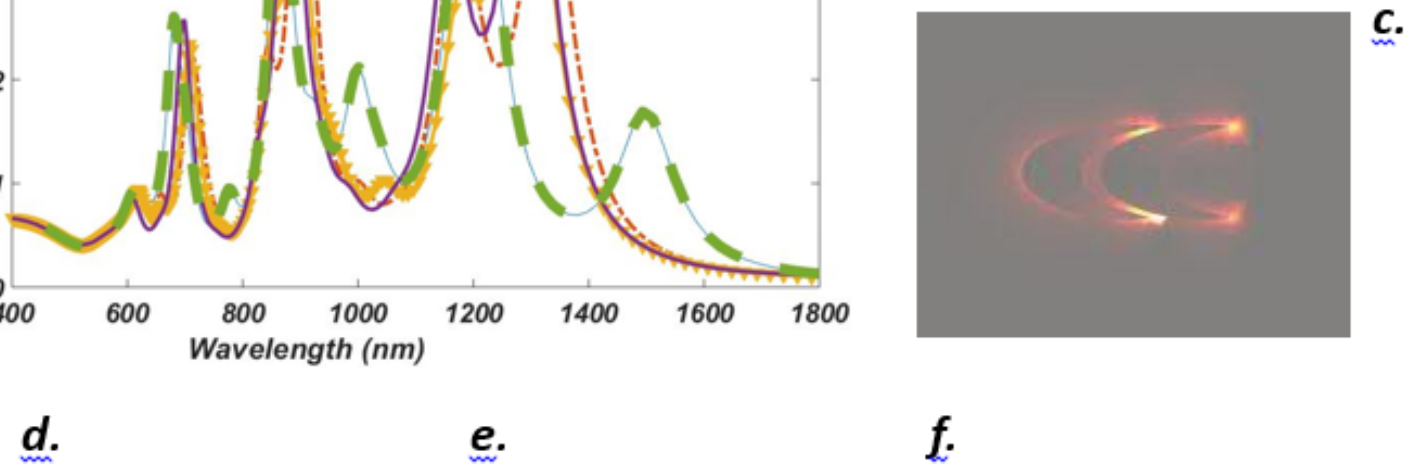

e.

f.
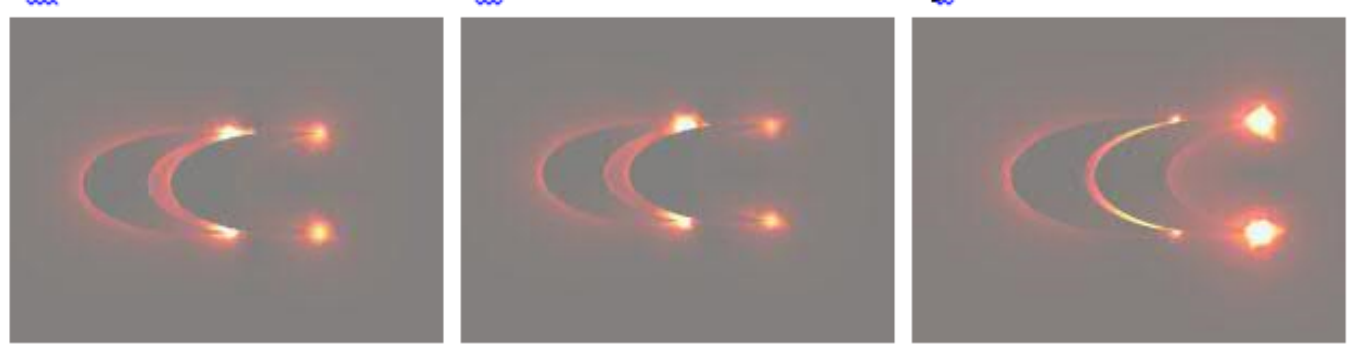

40

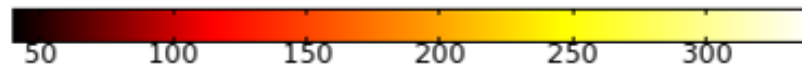

$\Delta 340$

Figure 6

(a) Extinction Cross-section of simple Nano-elliptical dimer (NED) type II for $x$ - polarization, (b-f) Near Field Enhancement (NFE) of type I NED for x-polarization luminance with separation between nano-ellipses (NE) S=3nm, 5nm, 7nm, 9nm and 11nm respectively 
a.

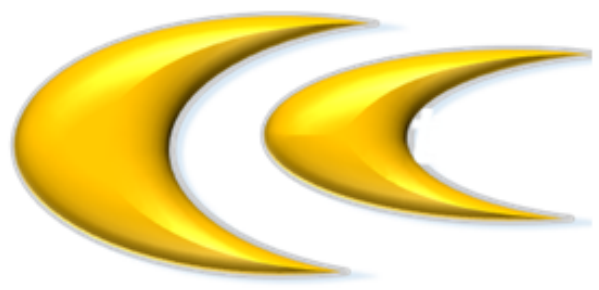

c.

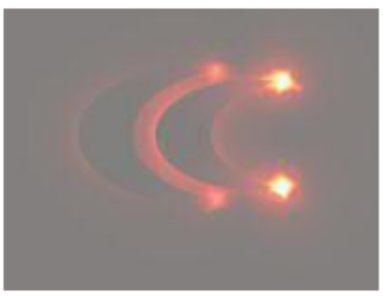

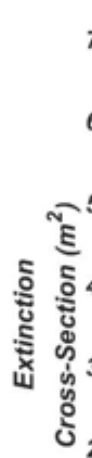

$7 \times 10^{-14}$

6

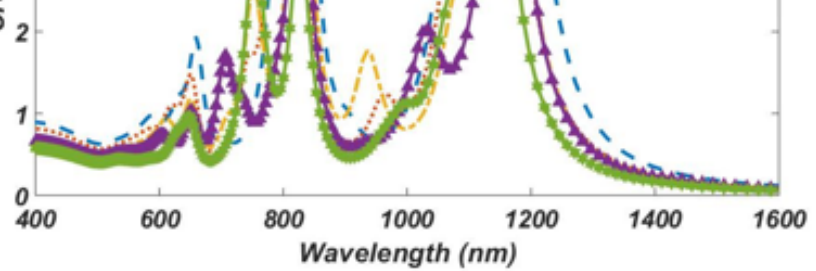

- Type IIA .... Type IIB - Type IIC -Type IID
- Type IIE

Wavelength $(\mathrm{nm})$

b.
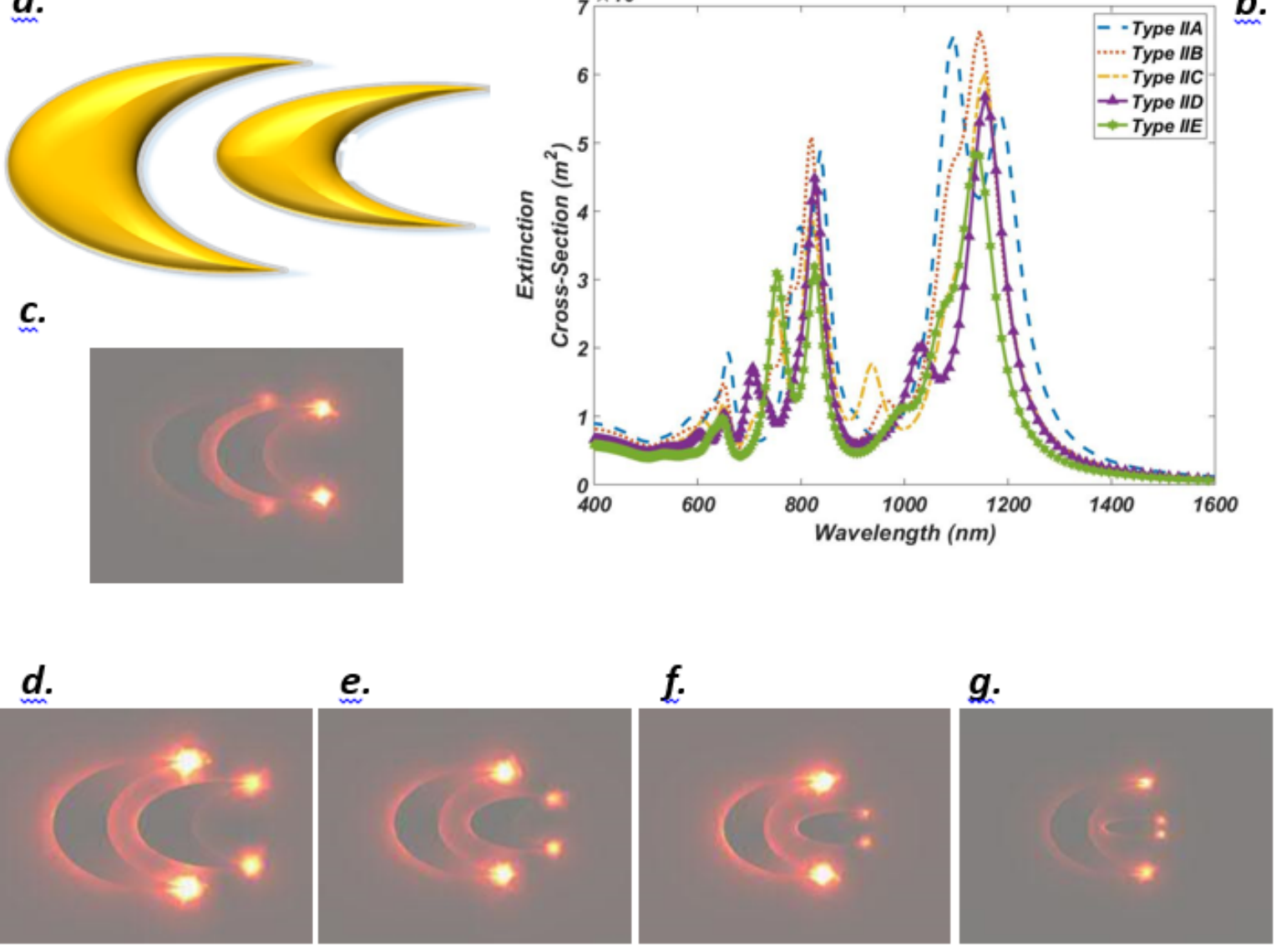

e.

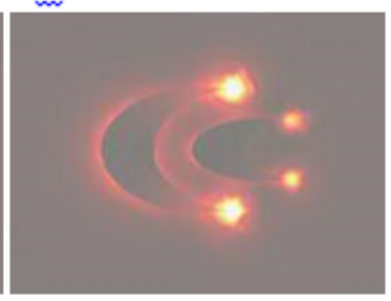

50 f.

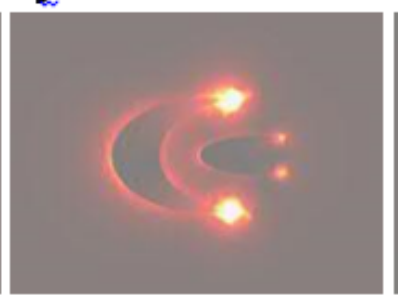

g.

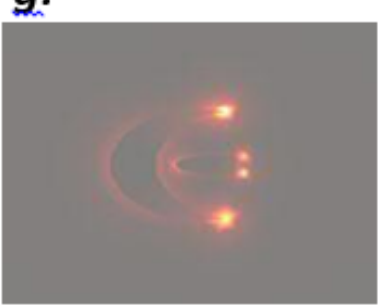

A 340

\section{Figure 7}

(a) Schematic of symmetry broken Type II NED (b) Extinction Cross-section of simple symmetry broken Nano-elliptical dimer (NED) type II for x-polarization, (c-g) Near Field Enhancement (NFE) of symmetry broken type II NED for x-polarization luminance. 


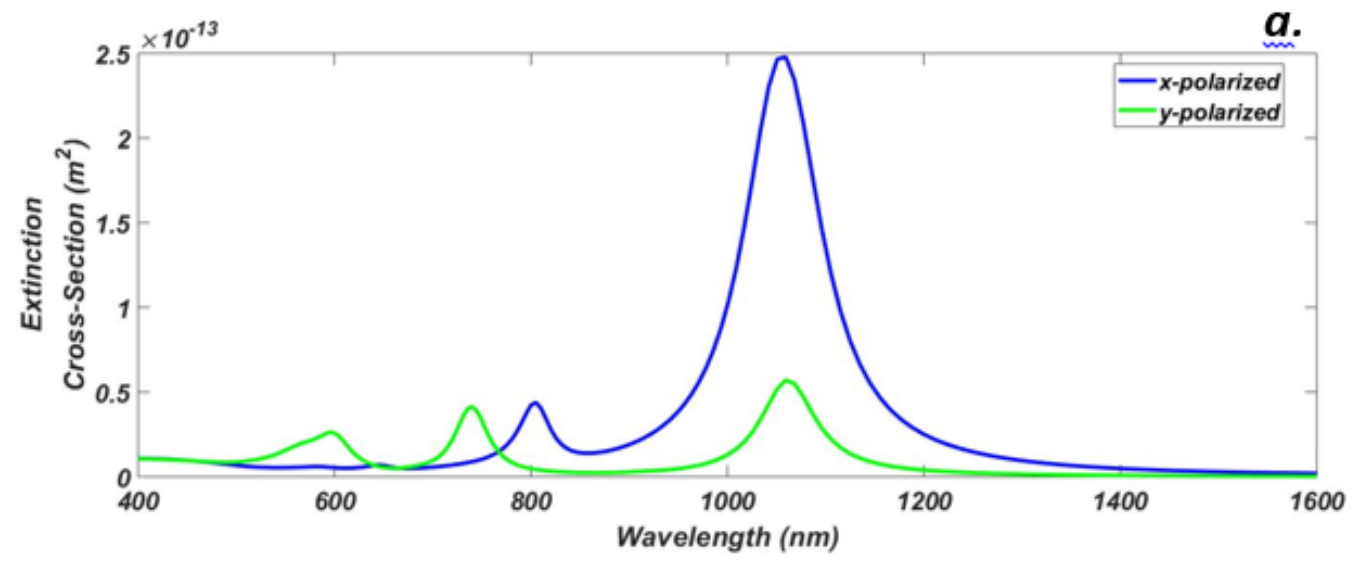

b.
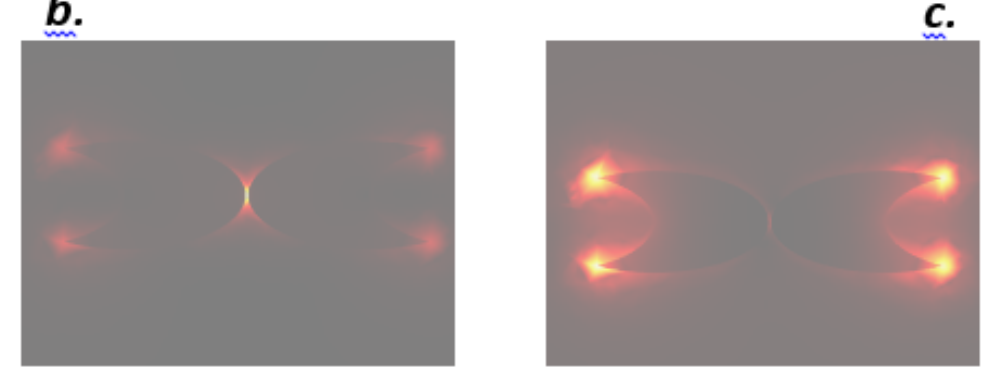

$\boldsymbol{\nabla} 40$

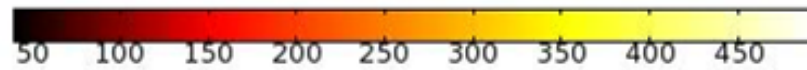

$\mathbf{\Delta} 460$

\section{Figure 8}

(a) Extinction Cross-section of simple Nano-elliptical dimer (NED) type III for $x$-y polarization, (b) Near Field Enhancement (NFE) of NED type III for x-polarization luminance, (c) Near Field Enhancement (NFE) of NED type III for y-polarization luminance 

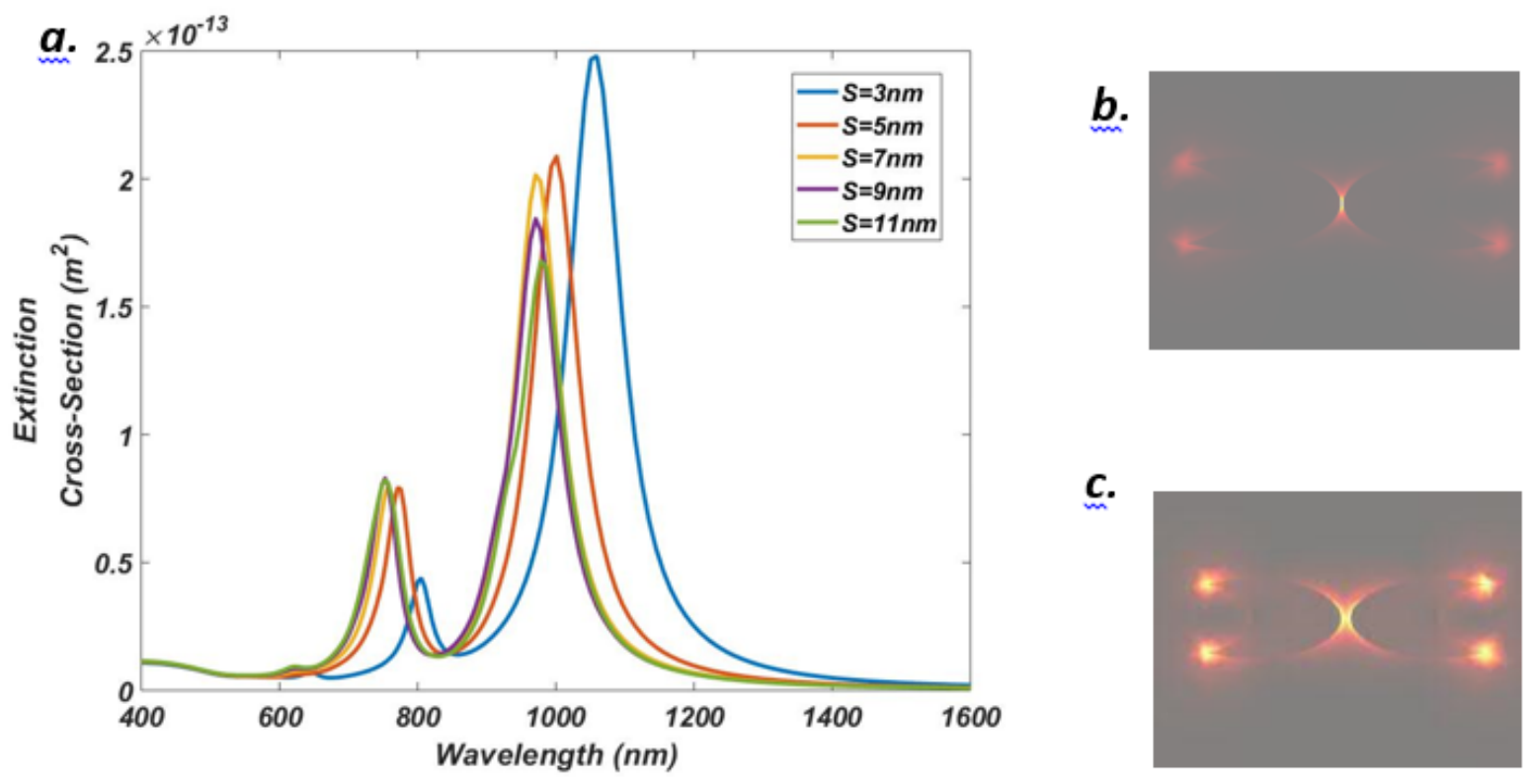

c.

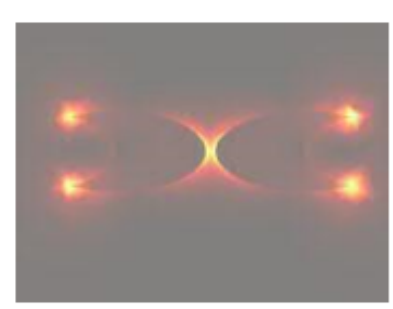

d.

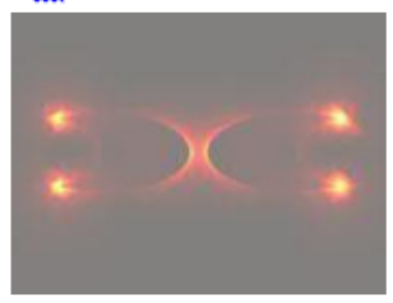

e.

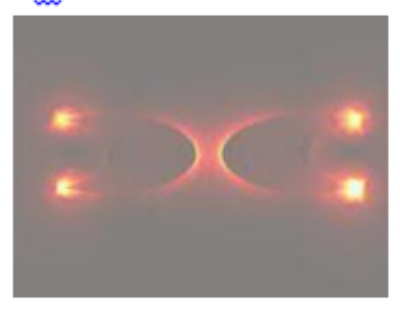

f.

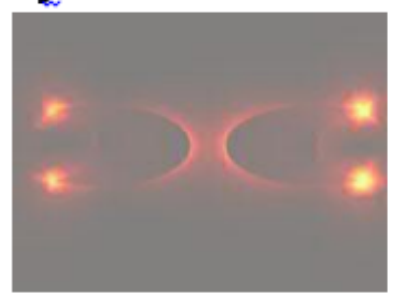

$\nabla 40$

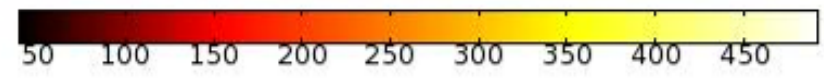

A 460

\section{Figure 9}

(a) Extinction Cross-section of simple Nano-elliptical dimer (NED) type III for $x$-polarization, (b-f) Near Field Enhancement (NFE) of type III NED for $x$-polarization luminance with separation between nano-ellipses (NE) $\mathrm{S}=3 \mathrm{~nm}, 5 \mathrm{~nm}, 7 \mathrm{~nm}, 9 \mathrm{~nm}$ and $11 \mathrm{~nm}$ respectively 
a.

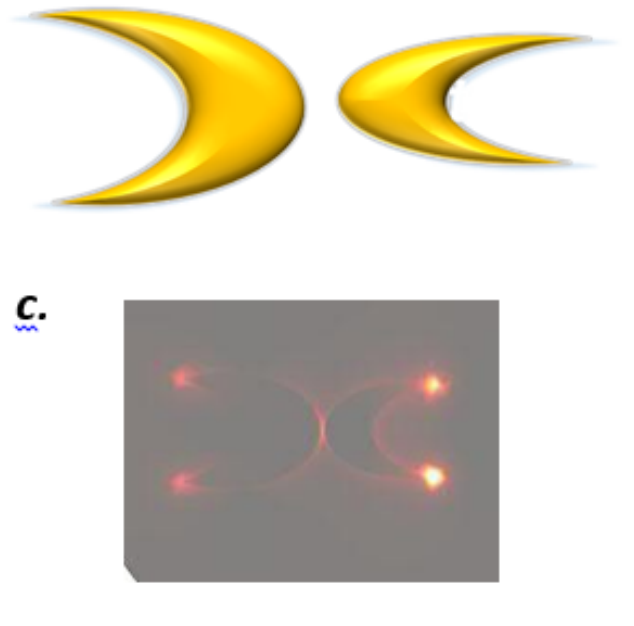

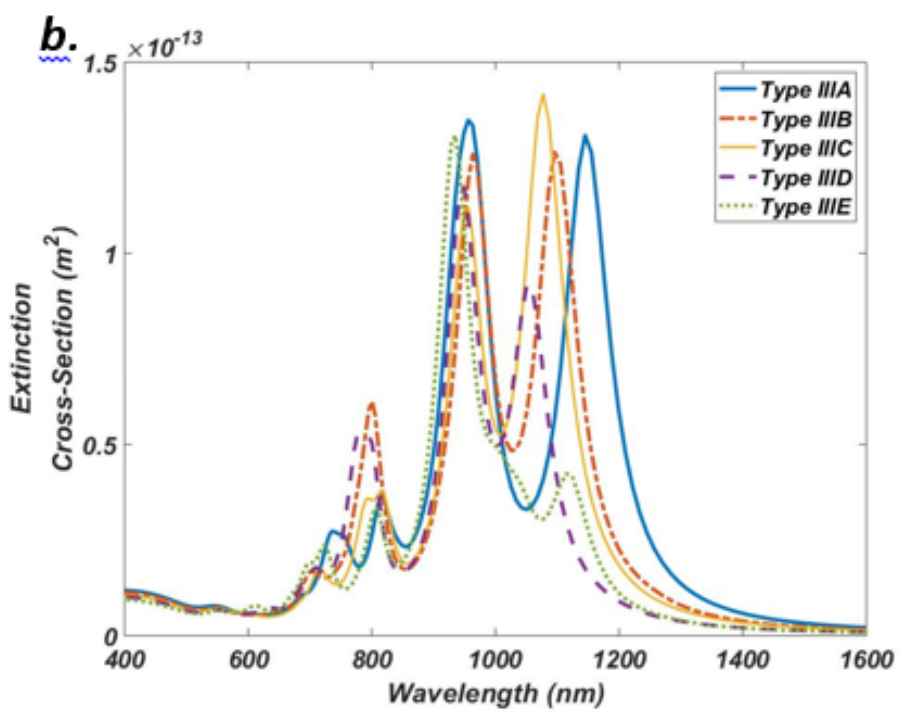

d.

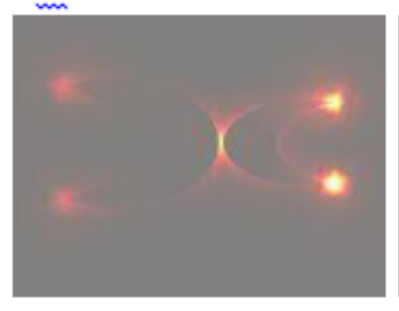

e.

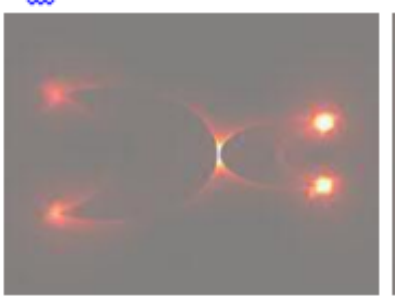

$f$.

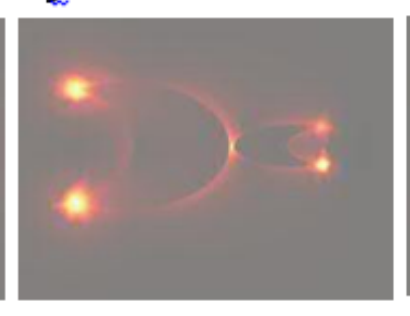

g.

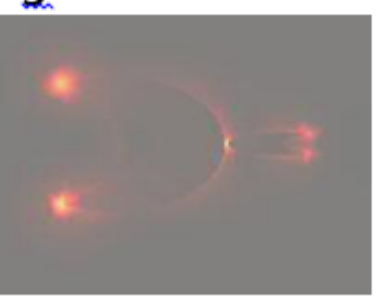

₹ 40

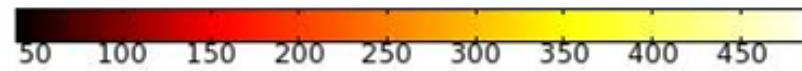

A 460

\section{Figure 10}

(a) Extinction Cross-section of simple Nano-elliptical dimer (NED) type III for $x$ - polarization, (b-f) Near Field Enhancement (NFE) of type III NED for $x$-polarization luminance with separation between nano-ellipses (NE) $S=3 n m, 5 n m, 7 n m, 9 n m$ and $11 \mathrm{~nm}$ respectively 